\title{
Optical inspection for quantification of decay on stone surfaces
}

\author{
P. Kapsalas ${ }^{\mathrm{a}}$, P. Maravelaki-Kalaitzaki ${ }^{\mathrm{b}}$, M. Zervakis ${ }^{\mathrm{a}, *}$, E.T. Delegou ${ }^{\mathrm{c}}$, A. Moropoulou ${ }^{\mathrm{c}}$ \\ ${ }^{a}$ Department of Electronics and Computer Engineering, Technical University of Crete, Chania 73100, Greece \\ ${ }^{\mathrm{b}}$ Ministry of Culture, 25th Ephorate of Prehistoric and Classical Antiquities, Chania 73100, Greece \\ ${ }^{\mathrm{c}}$ National Technical University of Athens, School of Chemical Engineering, Athens 15780, Greece
}

Received 19 April 2006; received in revised form 15 July 2006; accepted 16 July 2006

Available online 14 September 2006

\begin{abstract}
This paper examines a novel approach of corrosion damage analysis based on image processing for quantitative and qualitative evaluation of degradation effects on stone surfaces. This methodology can be applied in situ in association with a variety of nondestructive monitoring schemes, and on images acquired from several imaging modalities, capturing from micro- to macro-scale characteristics. Our analysis methodology was evaluated on three non-destructive monitoring techniques of cleaned and not cleaned stone surfaces, namely on digital camera, reflectography and fiber optic microscope images. Further to validating the potential of the various imaging modalities, the paper also assesses the corrosion rate and the efficiency of the recruited cleaning methods. The derived results are in accordance with chemical analyses revealing the deterioration patterns of the studied surfaces.
\end{abstract}

(C) 2006 Elsevier Ltd. All rights reserved.

Keywords: Morphological operations; Blob analysis; Statistical evaluation; Stone decay; Black crust

\section{Introduction}

Corrosion damage analysis and restoration has become an aspect of great concern during the last several years. Many researches have been developed aiming at studying the decay effects encountered on stonework and providing innovative tools for the removal of defects and the reconstruction [1]. The vast majority of the procedures that have been implemented so far though are based on corrosion estimation via the use of electrochemical methods. Such approaches are considered to be destructive for the stonework as they are based on the ablation of a specimen and subsequent application of a cleaning intervention [2,3]. Thus, the necessity of developing nondestructive procedures for estimating the corrosion effects has arisen. A pre-requisite for determining the decay patterns on stone surfaces is the comprehension of the corrosion mechanisms and the phenomenology that they arise.

The main constituent of black crusts observed at rainsheltered areas of monuments is due to sulfur dioxide

\footnotetext{
${ }^{*}$ Corresponding author. Tel.: + 302821037206.

E-mail address: michalis@danai.systems.tuc.gr (M. Zervakis).
}

$\left(\mathrm{SO}_{2}\right)$, which attacks calcite $\left(\mathrm{CaCO}_{3}\right)$ of calcareous stoneproducing gypsum $\left(\mathrm{CaSO}_{4} \cdot 2 \mathrm{H}_{2} \mathrm{O}\right)$ [4]. The formation of black crusts is associated with discoloration and development of a coating ranging from reddish-brown to brownblack color, depending on the exposure and chemical composition of the substrate. The most frequently encountered chemical constituents in black crusts are gypsum, calcite, silicates, potassium nitrate, metal oxides and numerous organic constituents in low concentration [2,5]. The thickness of crusts ranges from $100 \mu \mathrm{m}$ up to several $\mathrm{mm}$; successive sub-layers can be discriminated into the crust matrix, differing in texture and chemical composition. Black crusts do not form homogeneous varnished layers, but small black particles are sporadically located within the matrix of encrustation, as revealed by observations of thin sections of crusts under a polarizing microscope. These black particles are responsible for the coloration of the crust. Small white particles, often observed on the body of black crusts, are mainly associated with the presence of gypsum crystals and re-crystallized $\mathrm{CaCO}_{3}$.

Image processing (IP) techniques can be used for extracting information regarding the eroded areas in artworks, as indicated by previous works [6-10]. However, the investigation of such approaches is still in early stages. 
A survey of methods for quantifying the color alteration induced by weathering was conducted by Lebrun [6]. Pappas and Pitas [5] employed IP methods for the conservation of old paintings with the aim of diagnosing the deterioration effects and performing digital restoration. IP approaches were also applied to back-scattered electron images obtained with scanning electron microscopy-energy dispersive $\mathrm{X}$-rays analysis to identify and quantify salts and porosity with depth in porous media [8]. Furthermore, methods for characterizing the stone structure and detecting regions of material loss were developed in the study of Moltedo et al. [9] while Boukouvalas et al. in Ref. [10] introduce computer vision techniques for the detection and classification of mineral veins encountered on ceramic tiles surfaces. Despite the fact that image analysis as a diagnostic tool, in artwork conservation is still in early stages, it has been recruited in many applications of Materials' Science and particularly for the determination of decay effects on aerospace materials. Choi and Kim in Ref. [11] proposed a method of detecting corroded areas on aerospace materials and classifying these areas according to their characteristics. A similar study is also reported in Ref. [12] aiming at recognizing the various defect patterns of a cold mill strip with the aid of a binary decision tree.

The objective of the current paper is to investigate and assess the potential and the limitations of various monitoring methods (digital camera, reflectography in the visible band of spectrum (Vis) and fiber optics microscope, FOM) via IP techniques in nondestructive qualitative and quantitative evaluation of degradation on marble surfaces. The paper employs a computer-aided method for analysis of black and white particles observed on exposed marble decay areas. The employed approach combines operators that have been successfully applied to mammography image analysis, an area that poses similar peculiarities in both the structure of spots to be detected and the appearance of the background [13]. Similar algorithmic processes have been extensively applied to other medical applications, such as macular degeneration and analysis of computerized tomography magnetic resonance images (CT/MRI) images, with considerable success $[13,14]$.

To attain the desirable performance, we employ a multistage algorithm [15] for the estimation of location and rough extent of decay areas (providing qualitative measures) and the morphological analysis in terms of the shape of these areas (deriving quantitative measures). More specifically, our approach considers a Gaussian filtering process to determine the exact locations of prevalence of deterioration patterns and a morphological filtering process to derive more accurate shape information for such spots of deterioration. The results of both operators are fused through a conditional thickening operator with the aim of deriving both accurate location and shape information. Finally, the distinguished dark and bright spots are segmented to provide local intensity measurements related to the depth of deterioration.
The algorithm is applied to images obtained by different monitoring methods (digital camera, reflectography (vis), FOM) depicting a variety of degradation patterns. The particular imaging modalities were selected in order to assess how they converge in the determination of decay's extent and features. Reflectography in the visible and infrared spectral bands has been extensively used as a diagnostic tool of old paintings damage $[16,17]$. The monitoring system used in the current work was implemented by Balas et al. and provides information on the texture of the studied crusts [16].

Due to the quite general specifications employed in its design, the proposed algorithm can be applied for the evaluation of decay areas formed on a broad range of background surfaces. Further to its applicability on the FOM images it can also be used on images providing a more macroscopical description of the corrosion damage. Thus, it can be employed for estimating the degradation state of a stone material as it is illustrated by monitoring systems (digital camera and reflectography) providing macroscopic information, at the same or similar scale as the human eye. We can say that human inspection is often used for the evaluation of decay, over and above human inspection; however, our automated procedure is objective and reproducible and also derives numerical measures for quantifying the extent of decay. Besides the quantification of degradation effects, our analysis scheme can be used for an evaluation of the cleaning efficiency. The paper introduces several statistical measures comparing degraded versus non-affected stone regions, which reveal structural and intensity characteristics of the degradation process. Based on these measures we can assess not only the extent, but also the severity of degradation.

\section{Experimental setup}

The studied specimens correspond to marble surfaces where adjacent regions of cleaned and un-cleaned crusts prevail. The cleaning process was conducted by a Nd:YAG laser system used to partially remove the crust. The energy fluence of the Nd:YAG laser was fixed at $6.3 \mathrm{~J} / \mathrm{cm}^{2}$. Throughout the cleaning process, some parameters such as the laser pulses are modified resulting in the removal of crust layers differing in thickness. Each cleaned strip was obtained by increasing the number of laser pulses per spot from one up to six; a $40 \%$ area overlap was recorded between adjacent spots.

FOM, digital camera and a reflectography system were used to depict the degradation effects in the visible, infrared and near infrared bands of the spectrum. The images obtained by the predefined monitoring systems were used as input images in the automated method discussed in this paper. Aiming at providing information concerning the chemical composition of the detected decay areas, the segmented regions are classified into two broad categories: "black" and "white" particles. "Black particles" are associated with the presence of carbonaceous particles, 
alumino-silicates, dust, metal oxides and other pollutants embedded in the gypsum cavities. On the other hand, "white particles" represent decay patterns associated with gypsum crystals and re-crystallized $\mathrm{CaCO}_{3}$. The paper is focused towards investigating the potential and the limitations of the proposed algorithmic process in the estimation of decay encountered on images obtained by various monitoring systems. The degree of corrosion and the efficiency of the laser cleaning applications were assessed by considering the location of the decay patterns, their number, extent and thickness.

\section{Methodology for detection of decay areas}

The detection process aims at derving both coverage (percentage of area covered by spots) and morphological (number and exact area of spots) measures regarding decay areas. Thus, it addresses both accurate location estimation and efficient shape segmentation. In order to achieve these objectives operating on a locally varying background that often expresses the marble surface, the proposed approach is adaptive and combines several operators, each focusing on a particular objective. Its major components involve a Gaussian detector appropriate for location estimation and a morphological filter that allows more efficient shape preservation. The Gaussian detector is based on the difference of Gaussians (DoG) as a band pass filter that effectively enhances abnormal structures on a locally varying background [13-15], followed by twin thresholding for the segmentation of such structures. The morphological detector is based on the bot-hat filter for the enhancement of dark structures [18], followed again by twin thresholding for segmentation [19]. Comparing these two schemes, the Gaussian detector is capable of detecting the topology of deterioration patterns, but distorts their shape; the boundary of individual spots is over-smoothed. On the other hand, the morphological detector preserves the shape of deterioration patterns, but provides higher false-positive rates in detection. The combination (fusion) of the results of these two detectors via a conditional thickening operator guarantees low false-positive rate in detection and preserves shape attributes of the detected areas.

The conditional thickening operator denoted by $\otimes$ is applied on a spot $X$ relative to $Y$ with the pair of structuring elements $\left(M_{i 1}, M_{i 2}\right)$ as follows:

$$
\left(M_{i 1}, M_{i 2}\right) \otimes X Y=Y \cap\left(X \cup\left(\left(M_{i 1} \Theta X\right) \cap\left(M_{i 2} \Theta X^{\mathrm{C}}\right)\right)\right),
$$

where $X^{\mathrm{C}}$ denotes the complement of image $X$.

More specifically, a spot $X$ of the Gaussian detector is expanding up to the point that it reaches a neighboring spot or until it reaches the size of a co-located spot $Y$ detected by the morphological operator. The pair of structuring elements $M_{i 1}$ and $M_{i 2}$ controls the direction of expansion. To cover spatial expansion in many directions, we use eight pairs of such elements for either black or white spots. The first two pairs are given as

$$
\begin{aligned}
& \left(M_{11}, M_{12}\right)=\left(\left[\begin{array}{lll}
1 & 1 & 1 \\
0 & 0 & 0 \\
0 & 0 & 0
\end{array}\right],\left[\begin{array}{lll}
0 & 0 & 0 \\
0 & 1 & 0 \\
1 & 1 & 1
\end{array}\right]\right) \text { and } \\
& \left(M_{21}, M_{22}\right)=\left(\left[\begin{array}{lll}
0 & 0 & 0 \\
0 & 0 & 1 \\
0 & 1 & 1
\end{array}\right],\left[\begin{array}{lll}
0 & 0 & 0 \\
1 & 1 & 0 \\
0 & 1 & 1
\end{array}\right]\right)
\end{aligned}
$$

The remaining pairs are obtained from these matrix combinations through rotations every $90^{\circ}$. Finally, the conditional thickening operator is obtained as a combination of individual results for every pair $\left(M_{\mathrm{i} 1}, M_{\mathrm{i} 2}\right)$ :

$E=\bigcup_{i=1}^{8}\left(M_{i 1} M_{i 2}\right) \otimes X Y$.

The operator in Eq (2) forms the core of our detection approach, which employs the conditional thickening operator to combine and fuse the results of two individual detectors. For the decay areas, the patterns detected by the Gaussian detector are extended in space but the result is always intersected with the spots detected by the morphological detector. The intersection in each step preserves only spots that are co-located in both $X$ (after conditional thickening) and $Y$.

A related algorithmic approach had been previously employed by Dengler et al [18] in order to detect microcalcifications in mammogram images. However, the features of images in our application are quite different necessitating several changes to the method described in Ref. [18]. More specifically, the size of the Gaussian kernels as well as the structuring elements is chosen appropriately to reflect the individual characteristics of the studied problem. Furthermore, the locally varying contrast, noise and morphology of background in our application necessitate adaptive selection of parameters based on local features derived by the stone surface structure.

\section{Results}

The main aim of this section is to assess the potential and the limitations of the employed algorithmic approach both in corrosion damage diagnosis and in cleaning validation, using various non-destructive monitoring methods. The adopted approach is tested on a variety of images obtained through the three monitoring systems. In this paper though, we present results derived by studying the surface illustrated in Fig. 1(a). More specifically, the aforementioned surface is depicted via the FOM in 33 images and via the reflectography (vis) and digital camera by one image, respectively. These images are studied to validate the accuracy of each imaging modality and to estimate the degree to which result from each modality converge. Our evaluation is conducted in both qualitative and quantitative means. The qualitative validation is carried out by 

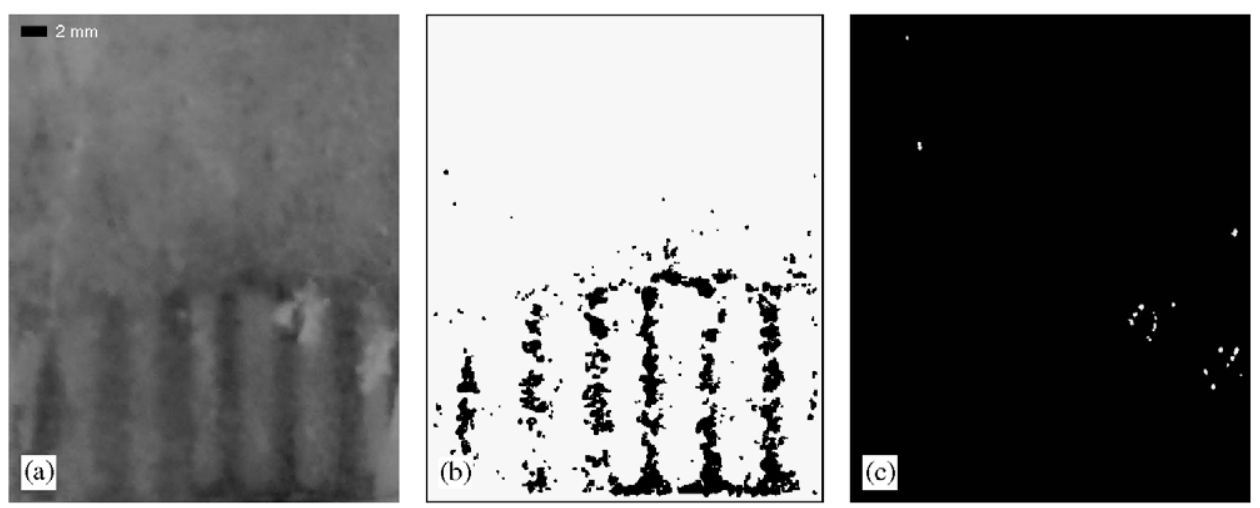

Fig. 1. (a) Stone material (monitored by a digital camera) demonstrating cleaned and un-cleaned stripes, (b) black particles detected, (c) white particles detected.

experts, by optically inspecting the detection results and the original images. Further to visual estimation, the detection results are also compared to assessments derived by the chemical study of the specimen under consideration. The above analysis is essential for validating the correctness of the detected areas in terms of their location of prevalence, as well as their size and shape characteristics. The statistical results obtained through the application of the automated approach exemplify the efficiency of this approach and elucidate the potential of the proposed monitoring systems as means of revealing and depicting corrosion effects.

\subsection{Qualitative results}

In this section, several results are presented demonstrating the effectiveness of the implemented approach under different monitoring systems. Fig. 1(a) illustrates the stone surface under consideration as it is depicted by the digital camera, while (b) and (c) shows the segmented black and white spots. As it is discussed in the experimental section, the stone specimen was partially cleaned by using a laser cleaning method with modified pulse intensities. In Fig. 1(a) we can observe the co-existence of untreated and treated stripes on the specimen; these can be discriminated by the color alterations, so that areas that exhibit a darker surface correspond to not cleaned regions.

As it is observed, the segmented degraded areas illustrate regions quite large in extent. The estimation process on the image from a digital camera does not reflect reliable information concerning the structure and the formation of black crusts. The latter can be further explained by considering the low resolution of the digital camera and the fact that it cannot sufficiently depict the structure of the crust, as it does not provide information related to texture characteristics. Thus, the segmentation procedure is carried out by considering only the color alteration, which is a secondary effect introduced by the presence of deterioration. Nevertheless, it should be noticed that the determination of the topology of treated and untreated regions is quite effective and in accordance to the experts judgment. For example, it is obvious that only a few black particles are segmented in areas depicting cleaned stripes. Regarding the segmentation of white particles, it should be noticed that the detected areas are associated with the ablation of stone material and do not reflect the existence of gypsum, re-crystallized $\mathrm{CaCO}_{3}$ or any other decay effect. The latter is associated to the fact that the inter-particle distance between the black spots in the crust matrix is not discernible under the digital camera monitoring. Thus, the prevalence of regions demonstrating bright abrupt changes is considered as white particles.

Reflectography is one of the non-destructive testing methods adopted in the analysis and diagnosis of artworks. The various modalities, infrared reflectography (IR), nearinfrared reflectography (NIR) and visible reflection (VR), allow carrying out tests using different options of illumination. Reflectography provides an effective tool to discover invisible details and texture irregularities. Fig. 2 depicts the same stone specimen as presented in Fig. (1), imaged via a reflectography system that acquire images operating in the spectral band of the visible light. The detected particles are depicted in Fig. 2 (b) and (c).

Optical inspection by the experts on Fig. 2 verifies that the topology of the detected black particles, their spatial distribution as well as their shape and size closely resemble to their own judgment of sporadic particle presence. A more dissect inspection of Fig. 2(c) though, reveals the detection of white decay spots even on treated regions. According to the experts' assessment, these spots are associated to regions of material loss. The above false positive induction illustrates the inability of the system to distinguish between areas where gypsum prevails and other areas where material loss occurs. This limitation arises from its low magnification as it becomes difficult to view the inter particle area between adjacent black spots and thus to accurately locate areas of gypsum or $\mathrm{CaCO}_{3}$ presence. A further explanation of the false positive detection of white spots is associated to the operation of the reflectography screening system. Thus, according to the literature [20], when visible light impacts on a black surface or an opaque surface (cavity), it is almost totally absorbed producing black-colored surface. In contrast, when the light impacts on a smooth and brilliant surface, it is reflected resulting in the depiction of white regions. In our 

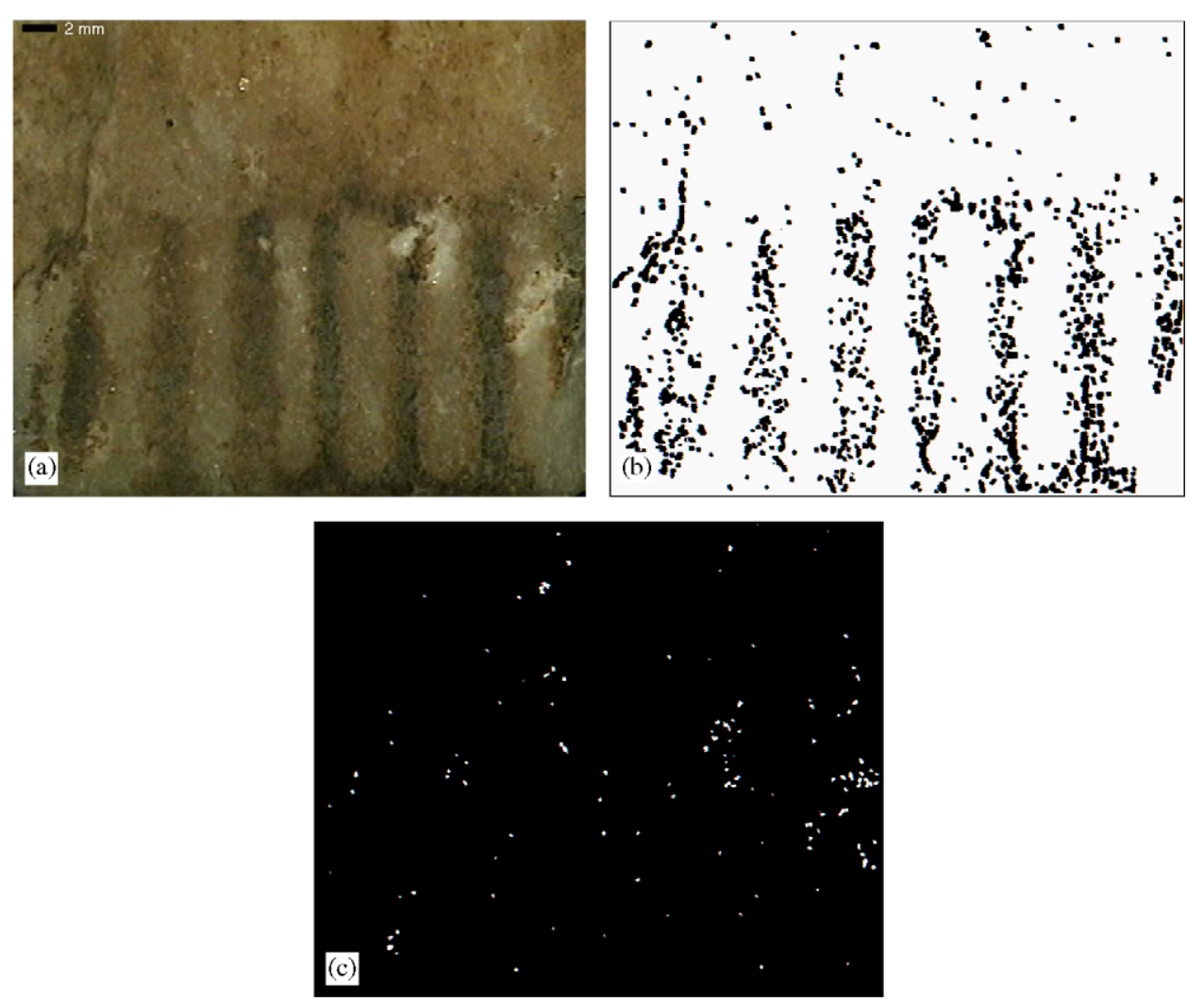

Fig. 2. (a) Stone material (monitored by a refectography system (vis)) demonstrating cleaned and un-cleaned stripes, (b) black particles detected, (c) white particles detected.

case, the areas corresponding to gypsum prevalence do not express smooth surfaces. In contrast, texture irregularities can be observed leading to diffusion of the impacting luminance and as a consequent, only a fraction of the light is reflected resulting to the illustration of white areas. This phenomenon provides a further explanation for the inaccurate segmentation of white particles.

As it is discussed in the introduction of this paper, one of our aims is to investigate the potential of using optical monitoring methods in the description of corrosion damage. To validate this potential, the results of such methods are compared with the results derived by imaging the same surface via a FOM system. Fig. 3(a) shows a part of the stone material of Fig. 2(a) monitored by the FOM system while (b) and (c) depict the black and white particles detected superimposed on the original image.

Experts' evaluation of the results reported in Fig. 3 reveals that the proposed automated method approaches accurately the topology of black spots while preserving information concerning their size and shape. Even the spatial distribution of deterioration patterns is in accordance with their own judgment of the sporadic presence of decay patterns into the matrix of encrustation.

\subsection{Quantitative results}

The corrosion effects encountered are subsequently quantified by measuring the number of spots, the percentage of area covered by such spots and their average size and spatial distribution. In order to make the results comparable regarding the spatial distribution of spots obtained through the various monitoring systems, we measure the mean number of black spots encountered per $\mathrm{cm}^{2}$ of the cleaned and un-cleaned areas of the stone material. Fig. 4 depicts the number of black particles detected on the surfaces studied in the previous subsection.

An obvious result that can be drawn from Fig. 4 is that the number of black patterns $/ \mathrm{cm}^{2}$ of stone surface encountered via the aid of the three monitoring methods is significantly reduced on cleaned areas. Moreover, it can be observed that FOM reveals a significantly higher number of detected black patterns. This can be explained by taking into account the high resolution provided by the FOM system, as well as its higher magnification range. In other words, FOM system demonstrates a better discern ability in detecting the individual patterns that prevail in the structure of black crusts while the other monitoring systems tend to merge adjacent degraded areas. The above assessment provides also a reason of the disability of the digital camera and reflectography to precisely determine the locations of white particles prevalence.

In order to overcome the different size of particles detected and rather focus on the overall effect on stone surfaces, Fig. 5 depicts the percentage of surface covered by black particles in the same cases as in Fig. 4. 

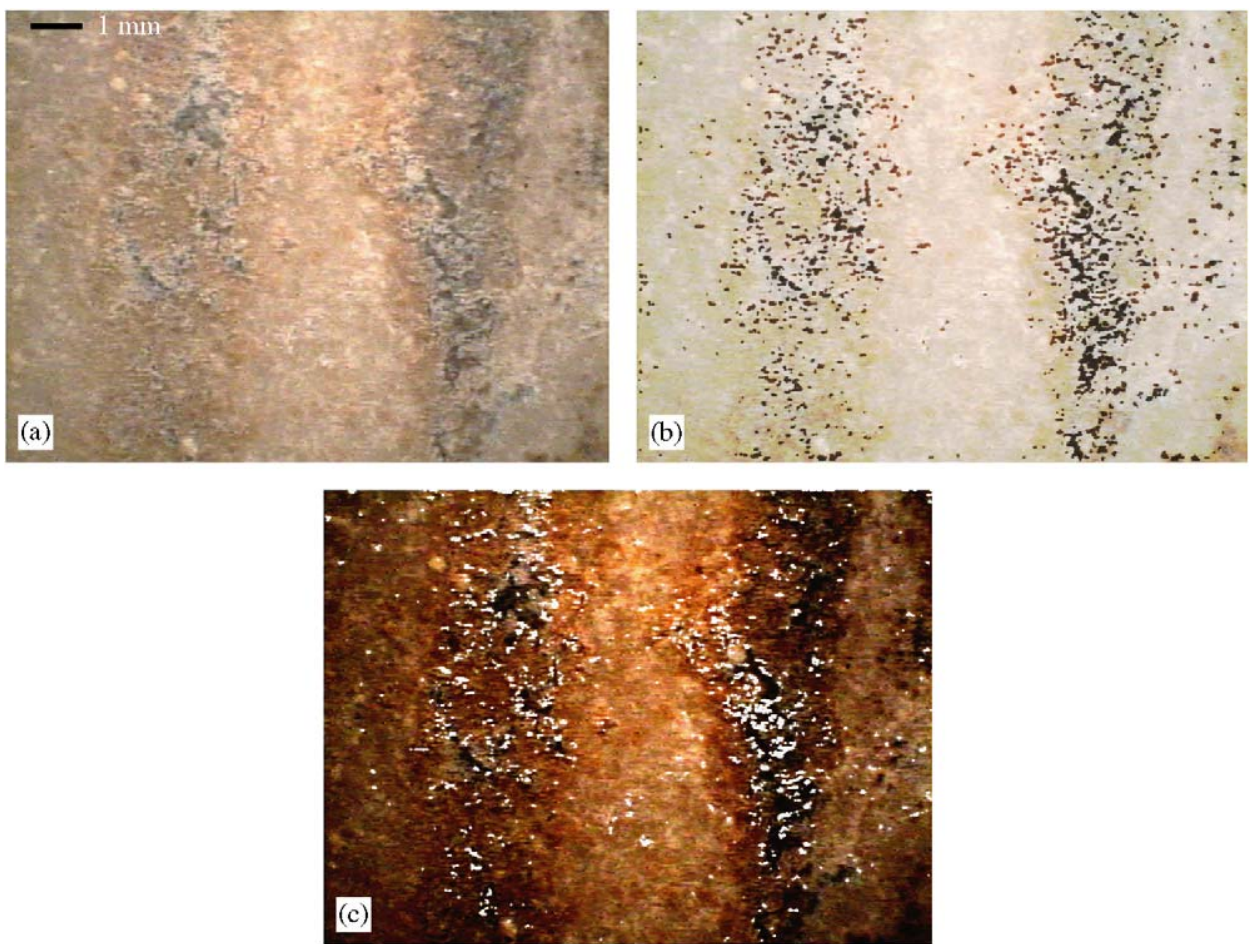

Fig. 3. (a) Stone material (monitored by a FOM system (magnification $25 \times$ )) demonstrating cleaned and un-cleaned stripes, (b) the detected black particles overlaid on the original surface, (c) the detected white particles overlaid on Fig. 3(a).

\section{Number of Black Paterns $/ \mathrm{cm}^{2}$ encountered throught the} different imaging methods

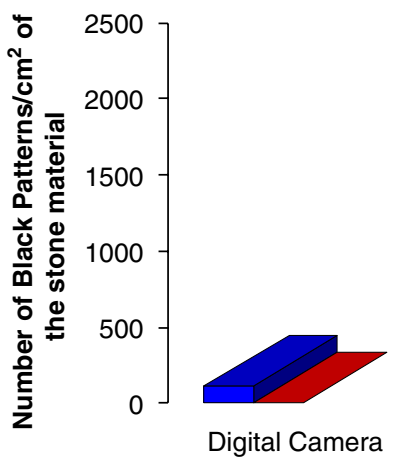

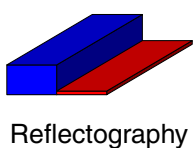

(vis)

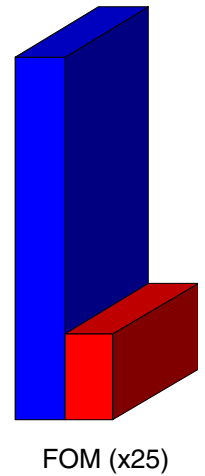

FOM (x25)

Monitoring Methods

- Uncleaned $\square$ Cleaned

Fig. 4. Number of black particles $/ \mathrm{cm}^{2}$ of stone surface as it was determined by the results of the different monitoring methods.

The results documented in Fig. 5, reveal that the fraction of the stone material covered by black particles is determined to be similar through the various monitoring systems. More specifically, the FOM system as well as the reflectography (vis) have only little divergence in the value of the percentage of stone covered by black particles (13.29\% and $13.47 \%$, respectively), while monitoring by the digital camera derives a fraction of the stone specimen equal to $11.42 \%$ to be covered by black patterns. By comparing together the results illustrated in Figs. 4 and 5, it becomes obvious that despite the greater number of black particles detected via the FOM system, the percentages of surface associated with corrosion damage tend to converge for all the three modalities. This observation reflects the potential of the FOM system to discriminate adjacent decayed areas while these areas tend to appear as larger merged regions when they are viewed by other imaging systems. Furthermore Fig. 5 reveals that cleaning 
achieves considerable elimination of black particles. The effect of cleaning is reflected by the significant reduction of both number and size of decay areas on the treated regions of the studied surfaces.

Another interesting quantitative measure concerning the size distribution of decay particles is summarized in Table 1. The distribution in terms of the median measure estimator rather than its mean measure is presented, since the actual size distribution on several images tested is heavily tailed. In particular, the measures computed are defined as follows:

- Median: The particle size that is greater than the $50 \%$ of the sizes detected on the image.

- Lower and upper quartiles: The particle sizes that are greater than $25 \%$ and $75 \%$ of sizes detected on the image, respectively.

The results reported in Table 1 indicate that the size distribution of black particles detected on not cleaned areas is spread to greater values than that detected on cleaned areas. This observation holds true for the surfaces monitored by the digital camera as well as for the surfaces monitored by the reflectography (vis) and the FOM system.

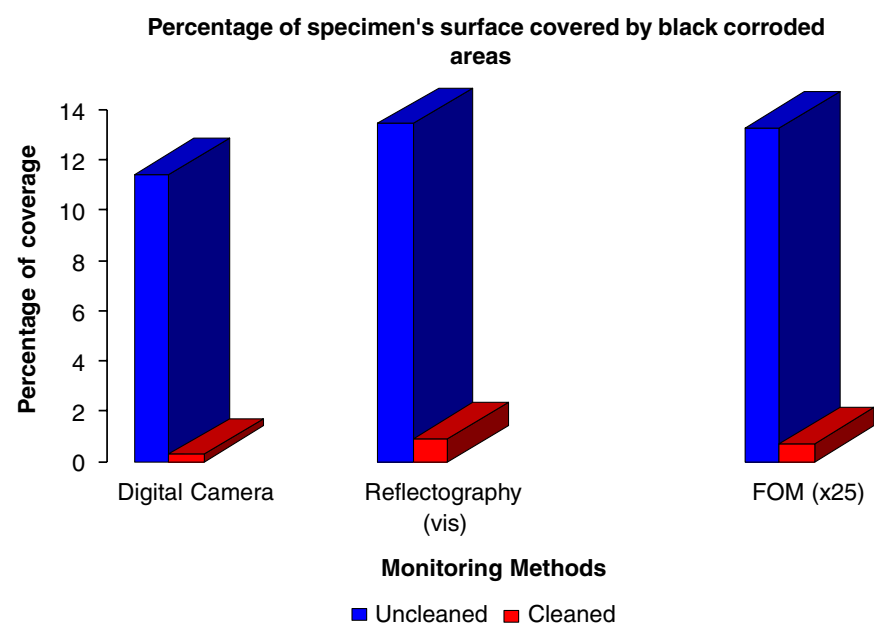

Fig. 5. Percentage of surface covered by black patterns, as it is determined through the various monitoring methods.
The above assessment indicates that the deterioration effects that cannot be removed by the cleaning process are, at least, reduced in extent. A significant point studying the results of Table 1 is the variation in the size distribution of black patterns through the different imaging systems. Thus, it can be observed that the detection on the FOM images segments corroded areas that correspond to smaller in extent surfaces on the stone material. This also supports the previous statements regarding differences in the ability of the employed monitoring systems to discriminate the prevalence of black particles onto the body of black crusts. In essence, the variation in the size of detected areas arises from the magnification of the systems considered, which reflects their individual potential to discern two contiguous decayed areas as two individual patterns, without merging them.

Up to this point, we consider only area measurements on the detected decay patterns. Intensity information from the original image on these patterns is also of great concern, since it relates with the depth of the crust accumulated on the surface. In this study, the aspect of crust thickness is approached in a rather qualitative point of view, in that darker formation implies more light absorption and, thus, thicker formation of black crust. Since the intensity distribution does not reflect heavy tails and resembles better the normal distribution, the reported measures present the mean and the upper and lower quartiles of the intensity over the particles of all images of the same type considered.

The results presented in Table 2 indicate the change in intensity distribution before and after chemical cleaning. It is verified that the employed automated approach derives intensity distributions shifted to lower values when applied to surfaces with black crusts of higher thickness (untreated areas). Moreover, after chemical cleaning the intensity distribution of the detected particles is increased, since such areas are diminished and appear brighter and less disturbing in a macroscopic point of view. This result also indicates that, even though cleaning does not completely eliminate all decay formations, it manages to reduce the thickness of remaining crust patterns relative to their original state. A comparison regarding the intensity distribution derived by the various monitoring methods

Table 1

Size distribution of decay patterns encountered on the studied surfaces

\begin{tabular}{llll}
\hline & $\begin{array}{l}\text { Lower quartile }\left(\mu \mathrm{m}^{2} \text { of }\right. \\
\text { stone surf })\end{array}$ & $\begin{array}{l}\text { Median }\left(\mu \mathrm{m}^{2} \text { of stone }\right. \\
\text { surf })\end{array}$ & $\begin{array}{l}\text { Upper quartile }\left(\mu \mathrm{m}^{2} \text { of }\right. \\
\text { stone surf) }\end{array}$ \\
\hline Digital camera image (not cleaned) & $363.93 \times 10^{2}$ & $647 \times 10^{2}$ & $1294 \times 10^{2}$ \\
Digital camera image (cleaned) & $242.62 \times 10^{2}$ & $364 \times 10^{2}$ & $485.25 \times 10^{2}$ \\
Reflectography image (not cleaned) & $186.27 \times 10^{2}$ & $479 \times 10^{2}$ & $878.16 \times 10^{2}$ \\
Reflectography image (cleaned) & $213.09 \times 10^{2}$ & $293 \times 10^{2}$ & $559.36 \times 10^{2}$ \\
FOM images (black spots) (not cleaned) & $26.83 \times 10^{2}$ & $47.71 \times 10^{2}$ & $74.46 \times 10^{2}$ \\
FOM images (black spots) (cleaned) & $20.92 \times 10^{2}$ & $34.74 \times 10^{2}$ & $52.11 \times 10^{2}$ \\
FOM images_(white spots) (not cleaned) & $23.21 \times 10^{2}$ & $40.37 \times 10^{2}$ & $66.05 \times 10^{2}$ \\
FOM images_(white spots) (cleaned) & $14.17 \times 10^{2}$ & $22.4 \times 10^{2}$ & $42.49 \times 10^{2}$ \\
\hline
\end{tabular}


reveals similar results with small variations, mainly arising from the different conditions of illumination of each modality.

As it was mentioned before, this work aims not only at studying the efficiency of the various monitoring modalities in defining corrosion damage, but also at assessing the extent and the severity of the degradation effects. Such a study is conducted by quantifying the deterioration state on parts of the stone surface that have been submitted to laser cleaning with different parameters. Fig. (6) depicts the studied stone material. As it can be observed, the treated and the untreated stripes are marked in the image. The $(\mathrm{T})$

Table 2

Intensity distributions of black particles detected at various surfaces

\begin{tabular}{lccc}
\hline & $\begin{array}{l}\text { Lower } \\
\text { quartile }\end{array}$ & Mean & $\begin{array}{l}\text { Upper } \\
\text { quartile }\end{array}$ \\
\hline $\begin{array}{l}\text { Digital camera image (not } \\
\text { cleaned) }\end{array}$ & 76 & 83.50 & 88 \\
$\begin{array}{l}\text { Digital camera image (cleaned) } \\
\text { Reflectography image (not }\end{array}$ & 100 & 103 & 124 \\
cleaned) & 59 & 78 & 82 \\
$\begin{array}{l}\text { Reflectography image (cleaned) } \\
\text { FOM images (not cleaned) }\end{array}$ & 72 & 98 & 88 \\
FOM images (cleaned) & 72 & 81 & 92 \\
\hline
\end{tabular}

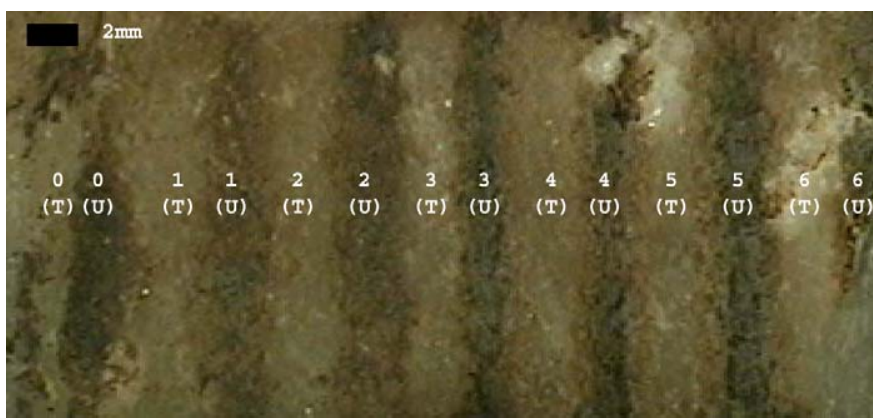

Fig. 6. The studied specimen having its treated and untreated stripes labeled. symbol indicates that the area was submitted to laser cleaning while $(\mathrm{U})$ indicates that no cleaning intervention was performed. The treated surfaces were cleaned by different laser-cleaning pulses resulting to more severe cleaning from left to right. We provide information concerning the cleaning efficiency at each application of the recruited intervention methods at each stone region through some statistical parameters. More specifically, the number of decay patterns detected per $\mathrm{cm}^{2}$, the percentage of the surface covered by decay as well as the distribution of decay pattern sizes encountered on the studied surfaces is used as means for quantifying the degradation state and estimating the cleaning efficiency at each region of application. Subsequently Figs. 7 and 8 illustrate the corrosion state encountered on the stripes defined in Fig. 6. In particular Fig. 7 presents the percentage of coverage of each of the studied stripes either cleaned or not cleaned by decay effects. The results are presented in logarithmic scale diagram where the data points illustrated at the left correspond to cleaned areas while those depicted at the right correspond to not cleaned areas. A diagram of similar structure is also presented regarding the number of decay patterns (Fig. 8). As it can be observed both of the diagrams provide information concerning both the treated and the untreated stripes in order to assess the crusts homogeneity prior to the cleaning as well as the cleaning efficiency at each adjustment of the cleaning parameters.

An initial study of Figs. 7 and 8 shows a significant reduction of the decay effects after the application of the cleaning intervention. This assessment holds true for both the cases of black and white particles encountered and is reflected on the number and the percentage of surface covered by corroded regions. A further observation of the results demonstrated in Figs. 7 and 8 also verifies that the treated stripes demonstrate a different degree of degradation. As we move from stripes 0 and 1 to the subsequent stripes, a subsistent elimination of corrosion occurs reflected by the reduction of number and percentage of black particles. This quantitative estimation is in accordance with the experts' judgment regarding the effects of

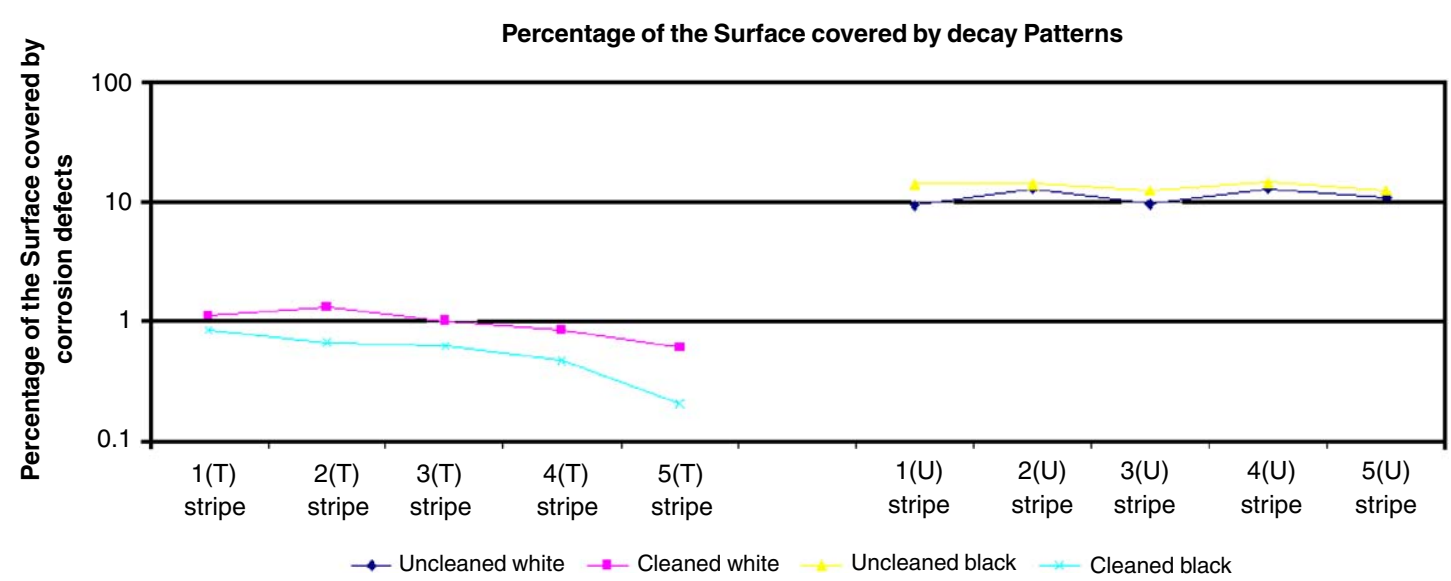

Fig. 7. Percentage of the stripes covered by corrosion damage both in cleaned (stripe $i_{\mathrm{a}} 1 \leqslant i \leqslant 5$ ) and the not cleaned areas (stripe $i_{\mathrm{b}} 0<i \leqslant 5$ ). 
Number of Decay Patterns Detected on the various stripes

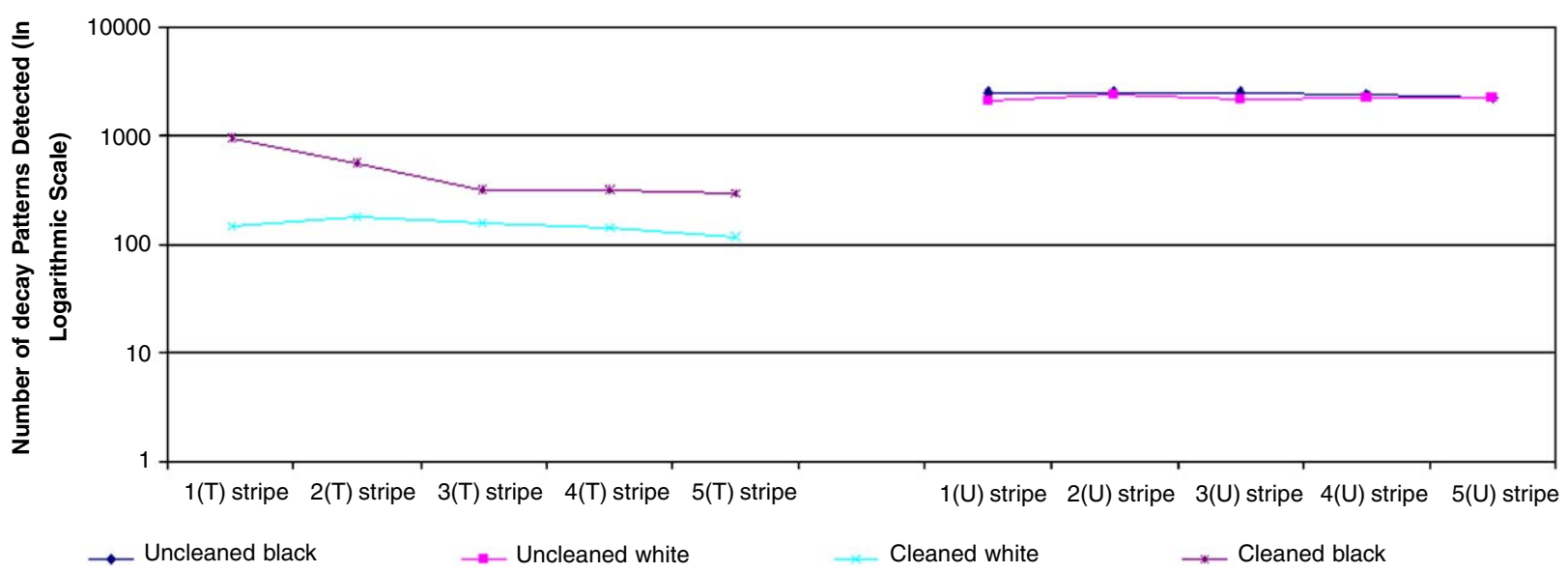

Fig. 8. Number of decay patterns encountered on the treated (stripe $i(\mathrm{~T}) 1 \leqslant i \leqslant 5$ ) and the untreated (stripe $i(\mathrm{U}) 0<i \leqslant 5$ ) areas of the stone specimen.

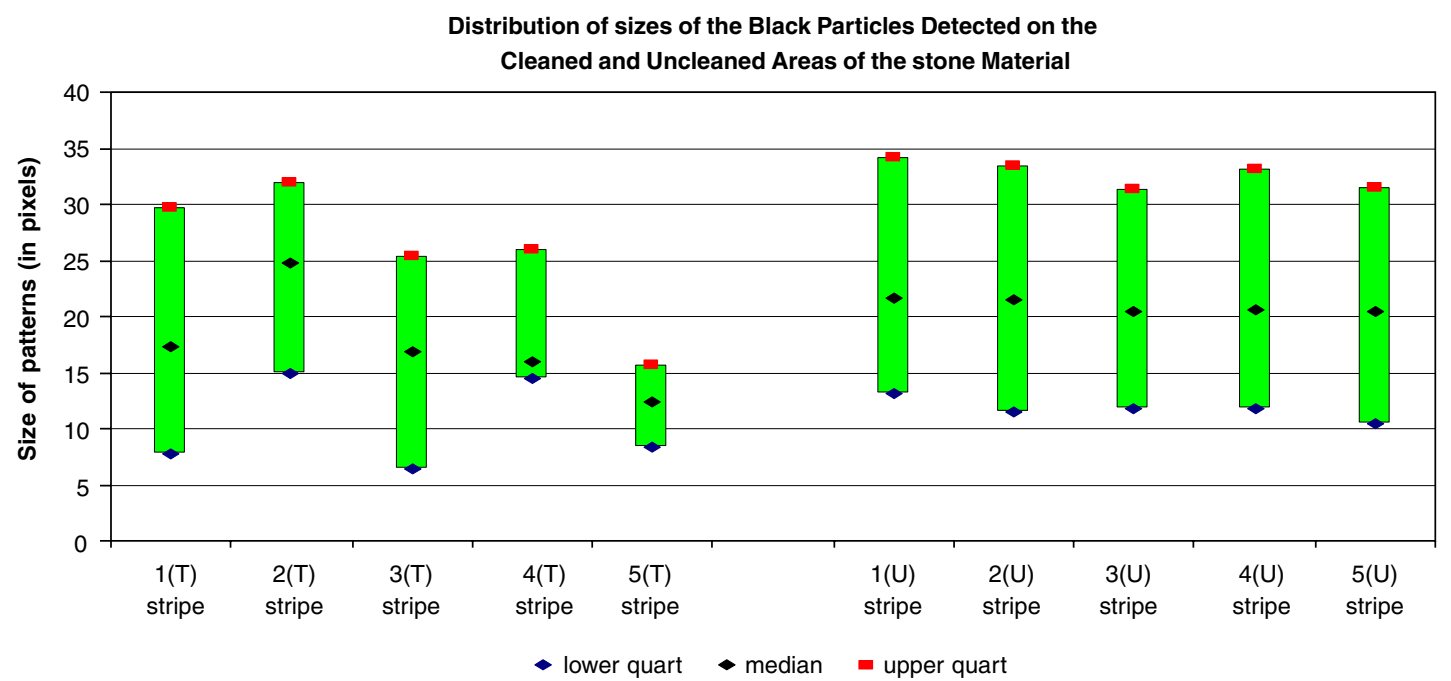

Fig. 9. Distribution of the surfaces of black spots encountered in both cleaned (stripe $i_{\mathrm{a}} 1 \leqslant i \leqslant 5$ ) and the not cleaned areas (stripe $i_{\mathrm{b}} 0<i \leqslant 5$ ).

the cleaning, as these stripes were cleaned by the application of increasingly higher laser pulses. Furthermore, it can be observed in the diagrams, that prior to the cleaning a homogeneous crust layer prevails on the stone surface. This is verified by the similar statistical measures employed to quantify corrosion. The size distribution of corrosion patterns is employed as a further means to estimate the cleaning effects or the degree of cleaning. In Fig. 9, we investigate any association present between the degree of cleaning and the extent of the decay patterns encountered.

Fig. 9 depicts a subsistent shifting of the size distribution to lower values, as we pass from stripes $1(\mathrm{~T})$ and $2(\mathrm{~T})$ to the consecutive stripes. The above diagram reveals that there might, indeed, exist an association between the size of the decay patterns and the cleaning state of the surface under consideration. In particular, it seems that the corrosion effects encountered on cleaned surfaces tend to be smaller in extent than those detected on untreated areas. An analogous observation can be drawn by observing the results concerning decay patterns on differently cleaned surfaces. Thus, we can detect larger in size patterns on surfaces less intensely cleaned. The fact that the distribution of decay patterns detected on the untreated regions display almost the same distribution of sizes supports the claim of homogeneous corrosion state before any treatment.

The above Figs. 7-9 illustrate the potential of the employed algorithmic approach to assess the corrosion rate as it can provide reliable results concerning even slight variation of the corrosion effects.

\section{Conclusions}

In this paper, we explore the potential of non-destructive inspection for studying environmental damage and assessing 
the effect of cleaning on stone monuments. We employ an algorithmic approach for non-destructive detection and segmentation of deterioration patterns on marble surfaces monitored via the aid of various imaging systems. Its design aims at localizing the decay areas while preserving their shape and extent. The proposed approach employs the conditional thickening operator to combine and fuse the results of two individual detectors, i.e. a Gaussian and a morphological detector, as to take advantage of the strong points of each one of them.

The set of images used to test the diagnostic procedure are obtained with the aid of a digital camera, a reflectography and a FOM system. Digital camera and reflectography (vis) provide macroscopic information, at the same scale as the human eye, while FOM provides more reliable information concerning the texture of the studied surface. The deterioration patterns detected by the proposed approach are described by the terms "black particles" and "white particles" while their prevalence is associated with the presence of carbonaceous particles in the body of black crusts.

The detection and segmentation of deterioration patterns provide the means for defining qualitative and quantitative measures associated with decay effects. The employed approach reveals different erosion characteristics depending on the imaging modality examined. Nevertheless, the percentage of degraded stone surface derived from our analysis scheme on any modality tested converges to almost the same amount. Thus, for degradation evaluation purposes even a simple camera imaging modality can be used with quite accurate results. Besides the quantification of degradation effects, our analysis scheme can be used for an evaluation of the cleaning efficiency. As it is assessed by the results derived, the number of the black particles is significantly lower on cleaned areas. Moreover, the proposed non-destructive approach enables measurements on the intensity distribution of decay, which is directly associated with the thickness of the crusts at these areas. The comparison of results derived by the different monitoring systems provides an overall assessment of the potential and the limitations of optical inspection (provided by the digital camera and the reflectography systems) in the reliable estimation of the corrosion damage.

\section{Acknowledgements}

This work was supported in part by the EC IST project BIOPATTERN, Contract no: 508803. The authors would like to acknowledge the valuable contribution of Prof. C. Balas (Department of Electronic and Computer Engineering, Technical University of Crete) for the Reflectography measurements and of Dr. A. Bakolas (School of Chemical Engineering, National Technical University of Athens) for the FOM measurements.

\section{References}

[1] Moropoulou A, Bisbikou K, Torfs K, Van Grieken R, Zezza F, Macri F. Origin and growth of weathering crusts on ancient marbles in industrial atmosphere. Atmos Environ 1998;32(6):967-82.

[2] Maravelaki-Kalaitzaki P. Black crusts and patinas on Pentelic marble from the Parthenon and Erechtheum (Acropolis, Athens): characterization and origin. Anal Chim Acta 2005;532(2):187-98.

[3] Maravelaki-Kalaitzaki P, Anglos D, Kilikoglou V, Zafiropulos V. Compositional characterization of encrustation on marble with laser induced breakdown spectroscopy. Spectrochim Acta Part B 2001; 56(6):887-903.

[4] Cammufo D. Acidification and its policy implications. Amsterdam: Elsevier; 1986.

[5] Jimenez SC. Deposition of airborne organic pollutants on historic buildings. Atmos Environ 1993;27(1):77-85.

[6] Lebrun V, Bonino E, Nivart JF, Pirard E. Development of specific acquisition techniques for field imaging-applications to outcrops and marbles. In: Proceedings of the international symposium of geovision on imaging applications in geology. Belgium: Liege; 1999. p. 165-8.

[7] Pappas M, Pitas I. Old painting digital color restoration. In: Noblesse workshop on non-linear model based image analysis. Scotland: Glasgow; 1998. p. 188-92.

[8] Cardell C, Yebra A, Van Grieken R. Applying digital image processing to SEM-EDX and BSE images to determine and quantify porosity and salts with depth in porous media. Microchim Acta 2002;140(1-2):9-14.

[9] Moltedo L, Mortelliti G, Salvetti O, Vitulano D. Computer aided analysis of buildings. J Cult Herit 2000;1(1):59-67.

[10] Boukouvalas C, et al. ASSIST: automatic system for surface inspection and sorting of tiles. J Mater Process Tech 1998;82(1-3):179-88.

[11] Choi KY, Kim SS. Morphological analysis and classification of surface corrosion damage by digital image processing. Corros Sci 2005;47(1):1-15.

[12] Kim K.M.: Design of a binary decision tree for recognition of the defect patterns of cold mill strip using generic algorithm. In: Innovations in applied artificial intelligence-proceedings of the 17 th international conference on industrial and engineering applications of artificial intelligence and expert systems, vol. 3029. Ottawa, Canada: IEA/AIE; May 17-20 2004.

[13] Shen L, Rangayyan RM. Detection and classification of mammographic calcifications. Int J Pattern Recogn Artif Intell 1993;7(6): 1403-16.

[14] Rapantzikos K, Zervakis M. Nonlinear enhancement and segmentation algorithm for the detection of age-related macular degeneration (AMD) in human eye's retina. In: Proceedings of the international conference on image processing. Greece: Thessaloniki; 2001. p. $1055-8$.

[15] Salfity MF, Kaufmann GH, Granitto P, Ceccatto HA. A computeraided diagnosis for automated detection and classification of clustered microcalcifications in mammograms. In: Proceedings of the third Simposio Argentino de Informática en Salud, 2000. p. 41-7.

[16] Georgiou S, Zafiropulos V, Anglos D, Balas C, Tornari V, Fotakis C. Excimer laser restoration of painted artworks: procedures, mechanisms and effects. Appl Surf Sci 1998;127-129:738-45.

[17] Balas C, Papadakis V, Papadakis N, Papadakis A, Vazgiouraki E, Themelis G. A novel hyper-spectral imaging apparatus for the nondestructive analysis of objects of artistic and historic value. J Cultural Heritage 2003;4(S1):330-7.

[18] Dengler J, Behrens S, Desaga JF. Segmentation of micro-calcifications in mammograms. IEEE Trans Med Imag 1993;12:634-42.

[19] Li H, Ray Lui KJ, Lo SB. Fractal modeling and segmentation for the enhancement of microcalcifications in digital mammograms. IEEE Trans Med Imag 1997;16:785-98.

[20] Obrutsky A, Acosta D. Reflectography, a NDT method for images diagnosis. In: Proceedings of the 16th world conference on nondestructive testing, Montreal, Canada; September 2004. 